\title{
LISTADO DE ESPECIES DE LA FAMILIA ORCHIDACEAE PARA EL ESTADO SUCRE, VENEZUELA
}

\author{
Carlos Leopardi ${ }^{1,2} \&$ Luis J. Cumana ${ }^{1}$ \\ ${ }^{1}$ Herbario Isidro Ramón Bermúdez Romero, Departamento de Biología, Universidad de Oriente, \\ Núcleo de Sucre, Cumaná, Sucre, 6101 Apdo. 245, Venezuela. \\ ${ }^{2}$ Autor para correspondencia: leopardiverde@gmail.com
}

Resumen. La familia Orchidaceae es uno de los grupos de Magnoliophyta de mayor distribución a nivel mundial, ocupan una amplia gama de nichos, desde el medio terrestre hasta las copas de los árboles (epífitas). Históricamente esta familia ha recibido mucha atención dada la belleza de las flores de algunos de sus miembros, lo que ha llevado con el tiempo a buscar el conocimiento, entre otras cosas, sobre su distribución; por lo que han aparecido listas de especies para varios lugares de América y el mundo. En Venezuela, exceptuando los tratamientos que a nivel general se ha dado a la familia, son escasos los estudios a nivel local y en particular en la cordillera de la costa; razón por la que en este trabajo se presenta una lista actualizada de las especies de Orchidaceae conocidas hasta el presente en la flora del estado Sucre. Para ello se revisaron entre los años 20062007 los herbarios VEN e IRBR, las bases de datos en línea de los herbarios K, MO, AMES, NY y F; aunado a ello, se realizaron exploraciones en 14 localidades del estado Sucre, distribuidas entre el extremo occidental de la Península de Araya hasta el extremo oriental de la Península de Paria, los alrededores de Cumaná, el Parque Nacional Mochima y parte del Macizo del Turimiquire. De lo anterior deriva una lista de 121 especies distribuidas en 63 géneros, los más importantes son: Epidendrum L. (16 spp.), Habenaria Willd. (8 spp.), Pleurothallis R. Br. s. 1. (7 spp.), Maxillaria Ruiz \& Pav. s. str. (4 spp.) y Scaphyglottis Poepp. \& Endl. (4 spp.). Adicional a éstas, existen 12 especies, señaladas en la literatura, de las que seis cuentan con respaldo de colector y número de colección. Del total de las especies referidas para el estado Sucre, 15 son mencionadas en el Libro Rojo de la Flora Venezolana; de éstas, dos están en peligro crítico (Cattleya gaskelliana Rchb. f. y Psychopsis papilio (Lindl.) H.G. Jones), una en peligro, dos vulnerables y las restantes en la categoría menor riesgo.

AвSTRACT. The family Orchidaceae is one of the most widespread groups of Magnoliophyta, they occur in a broad range of habitats, from the terrestrial habit to the treetops (epiphytes). Historically, this family had received a great deal of attention because of the beauty of the flowers of some species, which has lead to study its distribution; as a consequence, lists of species have been published for various places of America and the world. In Venezuela, except for treatments, of the family on a general level, there are few studies of local areas, and very little on the coastal cordillera; for that reason, we offer an updated list of the species of Orchidaceae so far know in the flora of Sucre state. To achieve this, the herbaria VEN and IRBR were studied during 20062007, and the on-line database of herbaria K, MO, AMES, NY and F were consulted. Field exploration was undertaken in 14 localities in Sucre state, divided between the Peninsulas of Araya and Paria, around of Cumaná and the Macizo del Turimiquire. As results, we present a list of 121 species of 63 genera. The most important are: Epidendrum L. (16 spp.), Habenaria Willd. (8 spp.), Pleurothallis R. Br. s. 1. (7 spp.), Maxillaria Ruiz \& Pav. s. str. (4 spp.) y Scaphyglottis Poepp. \& Endl. (4 spp.). Additionally, 12 species were mentioned in the literature, of which six have data (collector and collection number). Of the total of the species know from Sucre state, 15 was mentioned in the Red Book of the Venenezuelan flora, two of these are in critic danger (Cattleya gaskelliana Rchb. f. y Psychopsis papilio (Lindl.) H.G. Jones), one in danger, two vulnerable, the rest are in the category minor risk.

Palabras ClAVe / Key words: Orchidaceae, lista de especies, species checklist, Sucre, Venezuela 
La familia Orchidaceae es el grupo con la mayor diversidad entre las Magnoliophyta, con más de 19,000 especies (Judd et al. 1999). Esta familia se distribuye por todo el mundo, alcanzando su máxima diversidad en las regiones tropicales, especialmente en los andes americanos (Dressler 1981). Esta amplia distribución y diversidad se asocian a una alta plasticidad adaptativa que le ha permitido colonizar una variedad de nichos, desde el medio terrestre, incluyendo formas litófitas y rupícolas hasta especies de vida aérea (epífitas) en las copas de los árboles (Chase et al. 2003).

Históricamente, por la belleza de las flores de algunos de sus miembros, esta familia ha recibido gran atención; como muestra la publicación de monografías sobre el grupo durante los siglos XVIII--XXI (v. gr. Lindey 1830, Bateman 1874, Dressler 1981; Prigdeon et al. 2005); así, como la aparición de sociedades hortícolas que pagaban y pagan importantes sumas por estas plantas, lo que ha permitido financiar las expediciones de los llamados "cazadores de orquídeas", como Schomburgk, Linden, entre otros, que visitaban las tierras americanas en busca de estas plantas (Texera 1991, Romero 1999).

Este interés histórico por las orquídeas, ha tenido gran influencia en la elaboración de listados de orquídeas u orquideofloras para Brasil, Trinidad y Tobago, Chile, Guyana, Surinam, Guyana Francesa, Colombia, Cuba, Panamá, Costa Rica y Venezuela, entre otros (Cogniaux 1965, Schultes 1967, Lehnebach 2003, Funk et al. 2007, Huber et al. 1998); sin embargo, estos trabajos en su mayoría son de carácter general, siendo escasos los estudios para áreas específicas, como el elaborado en el Parque Nacional Manuel Antonio en Costa Rica (Populin 1998).

Venezuela no escapa a esta realidad, a nivel general los trabajos de mayor envergadura que se han realizado son los de Dunsterville y Garay (1959, 1961, 1965, 1966, 1972, 1976), Foldats (1969, 1970a,b,c,d) y Romero y Carnevali (2000); a nivel local, la zona que ha recibido la mayor atención ha sido Guayana, donde se han realizado una serie de estudios menores como el el reporte elaborado por Steyermark de las exploraciones para el Ptari-Tepuy, Roraima cerro Duida (Schweinfurth 1957), el informe sobre las orquídeas de Cerro Autana (Dunsterville 1975), la aclaratoria sobre el complejo de especies de Epidendrum nocturnum de la Guayana (Carnevali \& Romero 1996) y otros estudios mayores que llevaron a la publicación de la orquideoflora de la zona (Carnevali et al. 2003).

En el nororiente de Venezuela, la familia Orchidaceae ha recibido poca atención, a pesar de la importancia de ésta en los resultados de las exploraciones botánicas como las realizadas por Steyermark al cerro Turimiquire y la región oriental adyacente (Schweinfurth 1957, Steyermark 1966) y a la Península de Paria (Steyermark \& Agostini 1967), los resultados de Lárez (2003) para el Parque Nacional el Guácharo, entre otros.

En el estado Sucre, el interés sobre esta familia se ha incrementado en los últimos años, publicándose una lista preliminar de especies basada sólo en información bibliográfica (Leopardi \& Véliz, 2006) y algunas listas especificas locales, como las presentadas para el Parque Nacional Mochima (Leopardi et al. 2007, Cumana, en prensa). El objetivo de este trabajo es mostrar la lista actualizada de especies de la familia Orchidaceae para el estado Sucre, basada en la revisión de herbarios y en trabajo de campo.

\section{Metodología}

El listado presentado es el producto de la revisión, entre los años 2006 y 2007, de los Herbarios Isidro Ramón Bermúdez Romero (IRBR) y del Herbario Nacional de Venezuela (VEN); así, como de las bases de datos disponibles en línea de los herbarios Royal Botanical Garden Herbarium (K) (K) (http:// www.kew.org/herbcat); Missouri Botanical Garden Herbarium (MO) (http://www.mobot.org/mobot/ research/herbarium.shtml); Oakes Ames Orchid Herbarium (AMES) (http://asaweb.huh.harvard. edu:8080/databases/specimen_index.html); New York Botanical Garden Herbarium (NY) (http:// sciweb.nybg.org/science2/hcol/vasc/index.asp); Field Museum of Natural History (F) (http://emuweb. fieldmuseum.org/botany/detailed.php); demás, de una serie de exploraciones realizadas en 14 localidades del estado Sucre, distribuidas de la siguiente forma: Península de Araya y adyacencias (Campoma, Cariaco, Guacarapo, Guayacán), Península de Paria (Río El Pilar, Yaguaraparo), alrededores de Cumaná (Parque Nacional Mochima, El Tacal, San Juan de Macarapana, Río Brito, Humo Negro, Cerro Imposible), Macizo del Turimiquire (Catuaro, Las Pideras de Cocollar). En el apéndice 1 se muestra una selección de los ejemplares revisados y/o colectados. 
La colección de las muestras siguió el protocolo tradicional para el procesamiento de material de herbario (Lindorf et al. 1999), tomando de uno a tres individuos (dependiendo de la abundancia); así mismo, en caso de que el material estuviese en fenofase reproductiva, porciones de las inflorescencias fueron conservadas en una mezcla de formol, ácido acético y etanol (FAA) en proporción 1:1:3; en caso de que el material estuviese en fenofase vegetativa se tomó una parte y se mantuvo en condiciones de vivero hasta alcanzar la floración, colectándose luego material para herborizar y conservándose algunas flores en FAA. El material colectado está depositado en IRBR.

El listado de especies presentado se ajusta en forma general al sistema propuesto por Chase et al. (2003) y sigue, en forma amplia, la sinonimia propuesta en tropicos.org (http://www.tropicos.org) y por Govaerts (2005). Algunos casos especiales sensu stricto son: Cohniella Pfitzer y Lophiaris Raf. Los Maxillarinae siguen el tratamiento sugerido por Blanco et al. (2007).

\section{Resultados y discusión}

La familia Orchidaceae, en el estado Sucre, está representada por 121 especies, de 63 géneros, los más numerosos son: Epidendrum (16 spp.), Habenaria (8 spp.), Pleurothallis s. 1. (7 spp.), Maxillaria s. str. (4 spp.) y Scaphyglottis (4 spp.) (tabla 1). Al considerar la riqueza por zona, se encuentra que la más pobre es la Península de Araya y áreas adyacentes (11 spp.); mientras que, la zona más rica es la Península de Paria (83 spp.), seguida del macizo del Turimiquire (36 spp.) y los Alrededores de Cumaná (33 spp.).

Estos resultados se relacionan con lo que ha sido estimado para el país, siendo los géneros más numerosos a nivel nacional Epidendrum (162 spp.), Pleurothallis s.l. (125 spp.) y Maxillaria s. 1. (100 spp.) (Huber et al. 1998, Carnevali et al. 2007). La coincidencia en la dominancia genérica puede asociarse a la diversidad de ambientes presentes en el estado Sucre: arbustales xerófilos, sabanas, manglares, bosques tropófilos, bosques húmedos, entre otros. También es notable que estos géneros alcancen su máxima diversidad en el estado en las vertientes de la Península de Paria y del Macizo del Turimiquire (tabla 1), que son las zonas más frescas y húmedas, lo que coincide con el hecho de que esta familia tiende a ser más diversa en zonas con características similares a la andina, que es donde alcanza su máxima diversidad (Dressler 1981, Carnevali et al. 2007).

La pobreza de especies en la Península de Araya se debe, básicamente, a las escasas precipitaciones (son inferiores a $260 \mathrm{~mm}$ anuales) y a la acción del viento, que en las zonas de menores elevaciones, hacia la región occidental de la península, alcanza velocidad suficiente como para impedir el establecimiento no sólo de los representantes de esta familia, sino de casi todos los grupos vegetales (Cumana 1999)

Tres especies son endémicas para el estado Sucre (Acianthera pariaënsis (Carnevali \& G. A. Romero) Carnevali \& G. A. Romero, Epidendrum dunstervilleorum Foldats y Masdevallia irapana $\mathrm{H}$. R. Sweet). Por otro lado, en el Libro Rojo de la Flora Venezolana (Llamozas et al. 2003), se mencionan 15 de las especies reportadas para el estado Sucre, distribuidas de la siguiente forma: Cattleya gaskelliana Rchb. f. y Psychopsis papilio (Lind1.) H. G. Jones son señaladas como especies en peligro crítico de extinción, Chysis aurea Lindl. como especie en peligro y Encyclia cordigera (Kunth) Dressler y Huntleya lucida (Rolfe) Rolfe como especies vulnerables, las siete especies restantes aparecen bajo la categoría de menor riesgo (tabla 1).

Adicionalmente, en los trabajos de Steyermark (1966), Steyermark y Agostini (1967) y Foldats (1969, 1970b,c,d), son referidas seis especies con colector y número de colección: Cycnoches lodigessii Lindl (Moritz, 611); Dichaea pendula (Aubl.) Cogn. (J. Steyermark, 91932), Epidendrum cardioglossum Rchb. f. (J. Steyermark y cols. 62596), Gomphichis costaricensis (Schltr.) Ames (J. Steyermark y cols, 62517), Liparis neuroglossa Rchb. f. (J. Steyermark, 62645), Oncidium bicolor Lindl. (Dunsterville 378); sin embargo, estas exsiccata no fueron encontradas en los herbarios revisados.

Finalmente, otras seis especies son mencionadas, principalmente por Foldats (1970a, b, c, d), pero sin ningún soporte localizable, a saber: Aspasia variegata Lindl., Brassia caudata Lindl., Epidendrum purpurascens Focke, Lockhartia acuta (Lindl.) Rchb. f.), L. oblongicallosa Carnevali \& G.A. Romero y Pleurothallis testaefolia (Sw.) Lindl. Por otro lado, existe una serie de especies que han sido encontradas en la zona limítrofe con el estado Monagas (del lado de Monagas) que, potencialmente, también podrían 
TABLA 1. Lista de especies de la familia Orchidaceae presentes en el estado Sucre, Venezuela.

\begin{tabular}{|c|c|c|c|c|c|c|}
\hline ESPECIE & $\mathbf{A}^{\mathrm{a}}$ & $\mathbf{P}$ & $\mathbf{C}$ & $\mathbf{T}$ & $\mathbf{D R}^{\mathrm{b}}$ & $\mathbf{L}^{\mathbf{c}}$ \\
\hline Acianthera lanceana (Lodd. ex Lindl.) Pridgeon \& M. W. Chase & - & + & - & + & & $\mathrm{MR} / \mathrm{ca}$ \\
\hline Acianthera pariaënsis (Carnevali \& G. A. Romero) Carnevali \& G. A. Romero & - & + & - & - & ES & . \\
\hline Beloglottis costaricensis (Rchb. f.) Schltr. & - & + & - & - & & . \\
\hline Brassavola cucullata (L.) R. Br. & + & + & - & - & . & . \\
\hline Campylocentrum micranthum (Lindl.) Rolfe & - & + & + & + & . & . \\
\hline Campylocentrum schneeanum Foldats & - & + & - & - & $\mathrm{EC}$ & . \\
\hline Catasetum macrocarpum Rich. ex Kunth & - & + & + & - & & . \\
\hline Catasetum planiceps Lindl. & - & + & + & - & & . \\
\hline Cattleya gaskelliana Rchb. f. & - & - & - & + & EO & $\mathrm{CR}$ \\
\hline Caularthron bicornutum (Hook.) Raf. & + & + & - & - & . & . \\
\hline Caularthron bilamellatum (Rchb. f.) R. E. Schult. & + & + & + & - & & . \\
\hline Chysis aurea Lindl. & - & + & - & - & . & EN \\
\hline Cohniella cebolleta (Jacq.) Christenson & + & + & + & - & . & $\mathrm{MR} / \mathrm{ca}$ \\
\hline Cyclopogon elatus (Sw.) Schltr. & - & - & - & + & . & . \\
\hline Cyrtopodium willmorei Knowles \& Westc. & + & - & + & + & 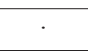 & . \\
\hline Dichaea hystricina Rchb. f. & - & - & - & - & & . \\
\hline Dichaea muricata (Sw.) Lindl. & - & - & - & - & $\cdot$ & . \\
\hline Dimerandra emarginata (G. Mey.) Hoehne & + & + & + & - & . & $\cdot$ \\
\hline Elleanthus arpophyllostachys Rchb. f. & - & + & - & - & $\mathrm{EC}$ & . \\
\hline Elleanthus furfuraceus (Lindl.) Rchb.f. & - & - & - & + & $\cdot$ & $\cdot$ \\
\hline Encyclia cordigera (Kunth) Dressler & + & - & + & + & & VU \\
\hline Encyclia isochila (Rchb. f.) Dodson & - & + & - & - & . & . \\
\hline Epidendrum anceps Jacq. & - & + & - & + & $\cdot$ & . \\
\hline Epidendrum cernuum Kunth & - & - & - & + & . & $\cdot$ \\
\hline Epidendrum ciliare var. squamatum Schnee & + & - & + & - & RV & $\cdot$ \\
\hline Epidendrum coronatum Ruiz \& Pav. & - & - & - & + & . & $\mathrm{MR} / \mathrm{ca}$ \\
\hline Epidendrum dunstervilleorum Foldats & - & + & - & - & ES & . \\
\hline Epidendrum ferrugineum Ruiz \& Pav. & - & + & - & + & . & . \\
\hline Epidendrum miserrimum Rchb. f. & - & + & - & - & & . \\
\hline Epidendrum pseudoramosum Schltr. & - & + & - & - & . & . \\
\hline Epidendrum purum Lindl. & - & + & - & - & . & $\cdot$ \\
\hline Epidendrum ramosum Jacq. & - & + & - & - & . & $\cdot$ \\
\hline Epidendrum rigidum Jacq. & - & + & + & + & & . \\
\hline Epidendrum rostratum Garay \& Dunst. & - & + & - & - & & $\cdot$ \\
\hline Epidendrum secundum Jacq. & - & + & - & + & & . \\
\hline Epidendrum strobiliferum Rchb. f. & - & + & - & - & . & . \\
\hline Epidendrum carpophorum Barb. Rodr. & - & + & - & + & . & . \\
\hline Epidendrum vincentinum Lindl. & - & + & - & - & . & $\mathrm{MR} / \mathrm{ca}$ \\
\hline Eulophia alta (L.) Fawc. \& Rendle & - & + & - & - & $\cdot$ & $\cdot$ \\
\hline Gongora quinquenervis Rchb. f. & - & - & + & + & . & . \\
\hline Govenia utriculata $(\mathrm{Sw}$.) Lindl. & - & - & + & + & . & . \\
\hline Habenaria alata Hook. & - & - & + & - & $\cdot$ & $\mathrm{MR} / \mathrm{ca}$ \\
\hline
\end{tabular}




\section{ESPECIE}

Habenaria bractescens Lindl.

Habenaria entomantha (Lex.) Lindl.

Habenaria heptadactyla Rchb. f.

Habenaria monorrhiza (Sw.) Rchb. f.

Habenaria obtusa Lindl.

Habenaria petalodes Lindl.

Habenaria trifida Kunth

Heterotaxis discolor (G. Lodd.) Ojeda \& Carnevali

Houlletia odoratissima Linden ex Lindl.

Huntleya lucida (Rolfe) Rolfe

Isochilus linearis (Jacq.) R. Br.

Jacquiniella globosa (Jacq.) Schltr.

Jacquiniella teretifolia (Sw.) Britton \& Wilson

Laelia undulata (Lindl.) L. O. Williams

Leochilus labiatus (Sw.) Kuntze

Lepanthes pariaënsis Foldats

Lepanthes wageneri Rchb. $\mathrm{f}$.

Lepanthopsis astrophora (Rchb. f.) Garay

Liparis nervosa subsp. nervosa Thunb.

Lophiaris lanceana (Lindl.) Braem

Lophiaris lurida (Lindl.) Braem

Macroclinium wullschlaegelianum (H. Focke) Dodson

Masdevallia irapana $\mathrm{H}$. R. Sweet

Masdevallia kyponantha H. R. Sweet

Masdevallia lansbergii Rchb. $\mathrm{f}$.

Maxillaria brachybulbon Schltr.

Maxillaria melina Lindl.

Maxillaria nasuta Rchb. f.

Maxillaria porrecta Lindl.

Microchilus paleaceus (Schltr.) Omerod.

Microchilus venezuelanus (Garay \& Dunst.) Ormerod.

Myoxanthus exasperatus (Lindl.) Luer

Notylia rhombilabia C. Schweinf.

Octomeria apiculata (Lindl.) Kuntze

Oeceoclades maculata (Lindl.) Lindl.

Oncidium klotzschianum Rchb. f.

Ornithocephalus bonplandii Rchb. f.

Ornithidium patulum (C. Schweinf.) M. A. Blanco \& Ojeda

Otoglossum globuliferum (Kunth) L. O.Williams \& M. W. Chase

Otoglossum scansor (Rchb. f.) Carnevali \& I. Ramírez

Pachyphyllum pastii Rchb. f.

Platystele oxyglossa (Schltr.) Garay

Platystele stenostachya (Rchb. f.) Garay

Pleurothallis discoidea Lindl.

\begin{tabular}{|c|c|c|c|c|c|}
\hline $\mathbf{A}^{\mathrm{a}}$ & $\mathbf{P}$ & C & $\mathbf{T}$ & $\mathbf{D R}^{\mathbf{b}}$ & $\mathbf{L}^{\mathbf{c}}$ \\
\hline- & - & + & - & . & . \\
\hline. & . &. & . & . &. \\
\hline - & - & + & - & . & . \\
\hline - & + & + & - & & $\mathrm{MR} / \mathrm{ca}$ \\
\hline - & - & + & - & & \\
\hline- & + & - & - &. & . \\
\hline- & - & + & - &. & . \\
\hline- & + & - & - & . & \\
\hline- & + & - & - & & \\
\hline- & + & - & - &. & VU \\
\hline- & + & - & + &. &. \\
\hline- & + & - & + &. &. \\
\hline- & - & - & + & . & . \\
\hline+ & - & + & - &. & \\
\hline- & - & - & + &. &. \\
\hline- & + & - & - & $\mathrm{RV}$ &. \\
\hline- & + & - & - & . & \\
\hline- & + & - & - & . & \\
\hline- & - & + & - & $\cdot$ & $\cdot$ \\
\hline+ & + & + & - &. & $\mathrm{MR} / \mathrm{ca}$ \\
\hline+ & + & + & + &. & . \\
\hline- & + & - & - &. & . \\
\hline- & + & - & - & ES & . \\
\hline- & + & - & - & . & . \\
\hline- & + & - & - & $\cdot$ & . \\
\hline- & + & - & - &. & \\
\hline- & + & - & - & . & . \\
\hline- & + & - & - & $\cdot$ &. \\
\hline- & + & - & + &. & . \\
\hline- & + & - & + & $\cdot$ & \\
\hline- & + & - & - & RV & . \\
\hline- & - & - & + &. &. \\
\hline. & $\cdot$ &. & $\cdot$ &. & . \\
\hline- & + & - & - & . & . \\
\hline- & + & + & + &. &. \\
\hline- & + & - & + &. & $\cdot$ \\
\hline- & - & + & - &. & . \\
\hline- & - & - & + & . & \\
\hline- & + & - & - &. & . \\
\hline- & + & - & - &. & \\
\hline- & - & - & + & $\cdot$ & . \\
\hline- & + & - & - &. & \\
\hline- & + & - & - & . & \\
\hline- & + & - & - &. & \\
\hline
\end{tabular}


TABLA 1. (continúa)

\begin{tabular}{|c|c|c|c|c|c|c|}
\hline ESPECIE & $\mathbf{A}^{\mathrm{a}}$ & $\mathbf{P}$ & $\mathbf{C}$ & $\mathbf{T}$ & $\mathbf{D R}^{\mathbf{b}}$ & $\mathbf{L}^{\mathbf{c}}$ \\
\hline Pleurothallis macrophylla Kunth & - & + & - & - & . & . \\
\hline Pleurothallis pruinosa Lindl. & - & - & + & - & . & $\cdot$ \\
\hline Pleurothallis revoluta (Ruíz \& Pav.) Garay & - & + & - & - & . & . \\
\hline Pleurothallis ruscifolia (Jacq.) R. Br. & - & + & - & - & . & $\cdot$ \\
\hline Pleurothallis aff. secunda Poepp. \& Endl. & - & - & + & - & . & $\cdot$ \\
\hline Polystachya foliosa (Hook.) Rchb. f. & - & + & - & + & . & . \\
\hline Prosthechea cochleata (L.) W. E. Higgins & - & - & - & + & . & $\mathrm{MR} / \mathrm{ca}$ \\
\hline Prosthechea aemula (Lindl.) W. E. Higgins & - & + & - & - & $\cdot$ & . \\
\hline Prosthechea livida (Lind1.) W. E. Higgins & - & - & + & + & . & $\cdot$ \\
\hline Psilochilus modestus Barb. Rodr. & - & + & - & - & $\cdot$ & $\cdot$ \\
\hline Psychopsis papilio (Lindl.) H. G. Jones & - & - & + & - & $\cdot$ & $\mathrm{CR}$ \\
\hline Psygmorchis glossomystax (Rchb. f.) Dodson \& Dressler & - & + & - & - & . & . \\
\hline Psygmorchis pusilla (L.) Dodson \& Dressler & - & - & + & - & . & . \\
\hline Rhetinantha notylioglossa (Rchb. f.) M. A. Blanco & - & + & - & - & $\cdot$ & $\cdot$ \\
\hline Rodriguezia lanceolata Ruiz \& Pav. & - & + & - & + & $\cdot$ & $\mathrm{MR} / \mathrm{ca}$ \\
\hline Sauvetrea aff. alpestris (Lindl.) Szlach. & - & - & - & + & $\cdot$ & $\cdot$ \\
\hline Scaphosepalum breve (Rchb.f.) Rolfe & - & + & - & - & $\cdot$ & $\cdot$ \\
\hline Scaphyglottis grandiflora Ames \& C. Schweinf. & - & + & - & - & $\cdot$ & $\cdot$ \\
\hline Scaphyglottis modesta (Rchb. f.) Schltr. & - & + & - & - & . & $\cdot$ \\
\hline Scaphyglottis prolifera Cogn. & - & - & - & + & . & . \\
\hline Scaphyglottis reflexa Lindl. & - & + & - & - & . & $\cdot$ \\
\hline Sobralia cf. ciliata (Presl) C. Schweinf. ex Foldats & - & + & - & + & . & $\cdot$ \\
\hline Sobralia aff. violacea Linden ex Lindl. & - & - & + & - & $\cdot$ & $\cdot$ \\
\hline Stelis argentata Lindl. & - & - & + & - & $\cdot$ & $\cdot$ \\
\hline Stelis papaquerensis $\mathrm{Rchb} . \mathrm{f}$. & - & + & - & - & $\cdot$ & $\cdot$ \\
\hline Stelis tristyla Lindl. & - & + & - & - & $\cdot$ & $\cdot$ \\
\hline Stenia pallida Lindl. & - & + & - & - & . & $\cdot$ \\
\hline Trichosalpinx ciliaris (Lindl.) Luer & - & + & - & - & . & $\cdot$ \\
\hline Trichosalpinx intricata (Lindl.) Luer & - & + & - & - & $\cdot$ & $\cdot$ \\
\hline Triphora gentianoides (Sw.) Nutt. ex Ames \& Schltr. & - & + & - & - & $\cdot$ & $\cdot$ \\
\hline Trizeuxis falcata Lindl. & - & + & + & + & $\cdot$ & $\cdot$ \\
\hline Vanilla pompona Schiede & - & + & + & - & . & $\mathrm{MR} / \mathrm{pm}$ \\
\hline Wullschlaegelia calcarata Benth. & - & + & - & - & $\cdot$ & . \\
\hline Xylobium pallidiflorum (Hook.) G. Nicholson & - & + & - & - & . & $\dot{0}$ \\
\hline Total de especies: & 11 & 83 & 33 & 36 & & \\
\hline
\end{tabular}

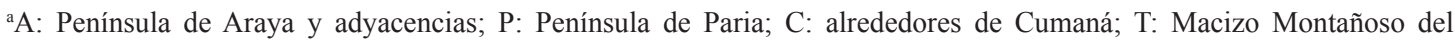
Turimiquire (sólo la sección que está en el estado Sucre).

bDR: Especies de distribución restringida; ES: endémica estado Sucre; EC: endémica Cordillera de la Costa; EO: endémica Cordillera de la Costa, región oriental; RV: restringida a Venezuela.

'L: estado de conservación según Llamozas et al. (2003); MR/ca: menor riesgo, casi amenazada; CR: peligro crítico; EN: en peligro; VU: vulnerable; MR/pm: menor riesgo, preocupación menor; $\because$ dato no disponible. 
estar en el estado Sucre, entre las que cabe destacar: Acianthera rubroviridis (Lindl.) Pridgeon \& M.W. Chase, Epidendrum subpurum Rchb. f., Ionopsis utricularioides (Sw.) Lindl., Mormodes buccinator Lindl., entre otras.

Agradecimientos. Los autores desean expresar su agradecimiento al Consejo de Investigación de la Universidad de Oriente (CI-2-010101-1224/05 y CI-2-010101-1374/07) y a PROVITA (2007-08) por el financiamiento otorgado. A la Dra Leyda Rodríguez (curadora de VEN) por el permitir la revisión de la colección de Orchidaceae allí depositada y por el préstamo de algunos de los ejemplares. A Carmen Malavé, Freddy Subero y Christian Marchán por toda la colaboración prestada durante el trabajo de campo. A José Imery y a los dos revisores anónimos por sus sugerencias y críticas al manuscrito. Al personal del IRBR por su colaboración durante la ejecución de este trabajo.

\section{LiTERATURA CITADA}

Bateman, J. 1874. A monograph of Odontoglossum. L. Reeve \& Co., Londres.

Blanco M., G. Carnevali, W. Whitten, R. Singer, S. Koehler, N. Williams, I. Ojeda, K. Neubig \& L. Endara. 2007. Generic realignments in Maxillariinae (Orchidaceae). Lankesteriana 7: 515-537.

Carnevali G. \& G. A. Romero. 1996. Orchidaceae Dunstervillorum VII: The Epidendrum nocturnum alliance in the Venezuelan Guayana and the Guianas. Lindleyana 11: 239-249.

Carnevali G., I. Ramírez-Morillo, G. Romero-González, C. Vargas, \& E. Foldats. 2003. Orchidaceae. Pp. 200-618 in: Steyermark, J. A., P.E. Berry \& B.K. Holst (Gen. eds.). P.E. Berry, B.K. Holst \& K. Yatskievych (vol. eds.). Fl. Venez. Guayana. Volume 7: Myrtaceae-Plumbaginaceae. Missouri Botanical Garden, St. Louis; Timber Press, Portland.

Carnevali G., G.A. Romero, E. Noguera. \& G. Gerlach. 2007. La familia Orchidaceae en Venezuela: diversidad y biogeografía. Memorias XVII Congreso Venezolano de Botánica: 21-23.

Chase M., J. Freudenstein, K. Cameron \& R. Barrett. 2003. DNA data and Orchidaceae systematics: a new phylogenetic classification. Pp. 69-89 in: Dixon, K., S. Kell, R. Barrett y P. Cribb (eds.). Orchid conservation. Natural History Publications. Kota Kinabalu.

Cogniaux A. 1965. Flora Brasiliensis. Orchidaceae. Vol. 3. Verlag von J. Cramer, New York, Estados Unidos.

Cumana L. 1999. Caracterización de las formaciones vegetales de la Península de Araya, estado Sucre,
Venezuela. Saber 11: 7-16.

Cumana L. 2008. Listado de las plantas vasculares del Parque Nacional Mochima, Venezuela. Ernstia 18: en prensa.

Dressler R. 1981. The Orchids: Natural History and Classification. Harvard University Press. Cambridge, Estados Unidos.

Dunsterville G. 1975. Orquídeas de la cima del cerro Autana. Acta Bot. Venez. 10: 251-262.

Dunsterville G. \& L. Garay. 1959. Venezuelan Orchids Illustrated. Vol. 1. André Deutsch Limited, Cambridge, Massachussetts.

Dunsterville G. \& L. Garay. 1961. Venezuelan Orchids Illustrated. Vol. 2. André Deutsch Limited, Cambridge, Massachussetts.

Dunsterville G. \& L. Garay. 1965. Venezuelan Orchids Illustrated. Vol. 3. André Deutsch Limited, Cambridge, Massachussetts.

Dunsterville G. \& L. Garay. 1966. Venezuelan Orchids Illustrated. Vol. 4. André Deutsch Limited, Cambridge, Massachussetts.

Dunsterville G. \& L. Garay. 1972. Venezuelan Orchids Illustrated. Vol. 5. André Deutsch Limited, Cambridge, Massachussetts.

Dunsterville G. \& L. Garay. 1976. Venezuelan Orchids Illustrated. Vol. 6. André Deutsch Limited, Cambridge, Massachussetts.

Foldats E. 1969. Flora de Venezuela. Orchideaceae. Tomo XV. I. Parte. Editorial Instituto Botánico. Caracas, Venezuela.

Foldats E. 1970a. Flora de Venezuela. Orchideaceae. Tomo XV. II Parte. Editorial Instituto Botánico. Caracas, Venezuela.

Foldats E. 1970b. Flora de Venezuela. Orchideaceae. Tomo XV. III Parte. Editorial Instituto Botánico. Caracas, Venezuela.

Foldats E. 1970c. Flora de Venezuela. Orchideaceae. Tomo XV. IV Parte. Editorial Instituto Botánico. Caracas, Venezuela.

Foldats E. 1970d. Flora de Venezuela. Orchideaceae. Tomo XV. V Parte. Editorial Instituto Botánico. Caracas, Venezuela.

Funk V., T. Hollowell, P. Berry, C. Kellof \& S. Alexander. 2007. Cheklist of the plants of Guiana Shield (Venezuela: Amazonas, Bolívar, Delta Amacuro; Guyana, Surinam, French Guiana). Contr. U.S. Natl. Herb. 55: 1-584.

Govaerts R., M. Campacci, D. Holland, P. Cribb, A. George, K. Kreuz \& J. Wood. 2005. "World Checklist of Orchidaceae". The Board of Trustees of the Royal Botanic Gardens, Kew. http://www.kew.org/wcsp/ monocots/, consultado el 21/06/2008. 
Huber O., R. Duno, R. Riina, F Staufer, L. Pappaterra, A. Jiménez, S. Llamozas \& G. Orsini. 1998. Estado actual del conocimiento de la flora de Venezuela. MARNR. Caracas.

Judd W., C. Campbell, E. Kellogg \& M. Stevens. 1999. Plant Systematic: A Phylogenetic Approach. Sinauer Associates. Massachussetts.

Lárez A. 2003. Angiospermas del Parque Nacional El Guácharo, estados Monagas y Sucre. Ernstia 13: 1-28.

Lehnebach C. 2003. Preliminary cheklist of the orchids of Chile. Bot. J. Linn. Soc. 143: 449-451.

Leopardi C. \& J. Véliz. 2006. Lista preliminar de la familia Orchidaceae para el estado Sucre, Venezuela. Saber 18: 95-103.

Leopardi C., J. Véliz. \& L. Cumana. 2007. Orquideoflórula preliminar del Parque Nacional Mochima, Venezuela. Memorias XVII Congreso Venezolano de Botánica: 763766.

Lindley J. 1830. The genera and species of orchidaceous plants. Ridgways. Londres, Inglaterra.

Lindorf H., L. Parisca \& P. Rodríguez. 1999. Botánica, clasificación, estructura y reproducción. Ediciones de la Biblioteca de la Universidad Central de Venezuela. Caracas, Venezuela.

Llamozas S., R. Duno de Stefano, W. Meier, R. Riina, F. Stauffer, G. Aymard O. Huber, \& R. Ortiz. 2003. Libro rojo de la flora venezolana. PROVITA - Fundación
POLAR - Fundación Instituto Botánico de Venezuela "Dr. Tobías Lasser". Caracas.

Pridgeon A., P. Cribb, M. Chase, \& F. Rasmussen. 2005. Genera Orchidacearum: Epidendroideae. Vol. 4. Oxford University Press, Oxford, Inglaterra.

Pupulin F. 1998. Orchid florula of Parque Nacional Manuel Antonio, Quepos, Costa Rica. Revista Biol. Trop. 46 (4): 961-1031.

Romero G. 1999. De viaje por las orquídeas. Analítica. http:/www.analitica.com/arte/1999.05/leer/pensar.htm, consultado el 21/06/2008.

Scheweinfurth, C. 1957. Orchidaceae. in: J. Steyermark (ed.). Contributions to the Flora the Venezuela. Field. Bot. 28 (4): 845-871.

Schultes R. 1967. Orchidaceae. Pp. 13-200 in: Flora of Trinidad y Tobago. Ministery of Agriculture, Industry and Comerce Trinidad and Tobago (ed.). Vol. 3, parte I. Government Printery Trinidad.

Steyermark J. 1966. El Cerro Turimiquire y la región oriental adyacente. Acta Bot. Venez. 1: 104-168.

Steyermark J. \& G. Agostini. 1967. Exploración botánica del Cerro Patao y zonas adyacentes a Puerto Hierro, en la Península de Paria, Estado Sucre. Acta Bot. Venez. 2: 7-80.

Texera Y. 1991. La exploración botánica en Venezuela (1754-1950). Fondo editorial Acta Científica Venezolana. Caracas. 
Apéndice 1. SElECCión Del MATERIAL EXAMINAdo

VENEZUELA. Sucre. Acianthera, A. lanceana: municipio Montes, Las Piedras de Cocollar, 31-03-2007, C. Leopardi et al. 103 (IRBR). A. pariänsis: municipio Valdez, Península de Paria, Cerro Patao, 19-07-1962, J. Steyermark y G. Agostini 91087 (VEN). Beloglottis, B. costaricensis: municipio Cajigal, Yaguaraparo, 18-02-2007, C. Leopardi et al. (IRBR). Brassavola, B. cucullata: municipio Cruz Salmerón Acosta, Guayacán, Península de Araya, 28-01-2006, C. Leopardi y J. Véliz 01 (IRBR). Campylocentrum, C. micranthum: municipio Cajigal, Yaguaraparo, 19-02-2007- C. Leopardi y F. Subero 99 (IRBR). C. schneeanum: municipio Mariño, Península de Paria, este de Cerro Humo, 24-02-1980, J. Steyermark y R. Liesner 121705 (VEN). Catasetum, C. macrocarpum: municipio Cajigal, Yaguaraparo, 18-02-2007, C. Leopardi et al. 76 (IRBR). C. planiceps: municipio Montes, Humo Negro, 12-10-2006, C. Leopardi y J. Véliz 49 (IRBR). Cattleya, C. gaskelliana: Entre Cerro El Diablo y La Trinidad, 1945, J. Steyermark 62765 (VEN). Caularthron, C. bicornutum: municipio Valdez, Península de Paria, noreste de Puerto Hierro, 05-09-1984, Milliken et al. 132 (VEN). C. bilamellatum: municipio Cajigal, Yaguaraparo, 17-02-2007, C. Leopardi et al. 68 (IRBR). Chysis, C. aurea: municipio Mariño, Península de Paria, Río Grande, 02-011981, J. Steyermark S/N (VEN). Cohniella, C. cebolleta: municipio Sucre, Vega grande, Parque Nacional Mochima, 22-01-1994, N. Ortega y N. Medina 70 (IRBR). Cyclopogon, C. elatus: municipio Montes, Las Piedras de Cocollar, 0204-2007, C. Leopardi et al. 129 (IRBR). Cyrtopodium: C. willmorei: Sabanas entre Sucre y Monagas, 28-04-2007, C. Leopardi y J. Véliz 140 (IRBR). Dichaea, D. hystricina: Sucre sin localidad exacta, S/F, Sin colector $S / N$. (IRBR). D. latifolia var longa: Sucre sin localidad exacta, S/F, Sin colector $S / N$. (IRBR). Dimerandra, D. emarginata: municipio Benítez, Río El Pilar, 06-05-2007, C. Leopardi y C. Malavé 141 (IRBR). Elleanthus, E. arpophyllostachys. municipio Mariño, Península de Paria, entre Los Pocitos y La Roma, 11-02-1966, J. Steyermark y M. Rabe 96345 (VEN). E. furfuraceus: municipio Montes, Las Piedras de Cocollar, 02-04-2007, C. Leopardi et al. 135 (IRBR). Encyclia, E. cordigera: municipio Cruz Salmerón Acosta, Península de Araya, Guarapo-Oturo, 15-12-1984, M. y W. Lampe 28 (IRBR). E. isochila: municipio Mariño, Península de Paria, Las Melenas, 01-12-1979, J. Steyermark y $R$. Liesner 120828 (VEN). Epidendrum, E. anceps: municipio Montes, Las Piedras de Cocollar, 01-04-2007, C. Leopardi et al. 121 (IRBR). E. carpophorum: municipio Montes, Macizo del Turimiquire, (25-31)-03-1995, L. Cumana et al. 6202 (IRBR). E. cernuum: municipio Montes, Cara norte del Cerro Turimiquire, cerca de la cumbre, (06-08)-05-
1945, J. Steyermark 62596 (VEN). E. ciliare var. Squamatum: municipio Cruz Salmerón Acosta, Laguna de Chacopata, , 18-12-2006, C. Leopardi y J. Véliz 57 (IRBR). E. coronatum: municipio Benítez, Río El Pilar, Hacienda El Otro Lado, 06-05-2007, C. Leopardi y C. Malavé 151 (IRBR). E. dunstervilleorum: municipio Mariño, Península de Paria, Cerro Humo, 03-03-1966, J. Steyermark 94972 (VEN). E. ferrugineum: municipio Mariño, Península de Paria, cerca de Río Seco, 11-08-1967, V. Carreño $S / N$ (VEN). E. miserrimum: municipio Mariño, Península de Paria, Cerro Humo, entre Los Pocitos y la Roma, 11-081966, J. Steyermark y M. Rabe 96347 (VEN). E. pseudoramosum: municipio Mariño, Cerro Humo, S/F, J. Steyermark y M. Rabe 96325 (VEN). E. purum: municipio Mariño, Península de Paria, Manacal, 20-02-1967, V. Carreño $S / N$ (VEN). E. ramosum, Sucre, S/F, J. Steyermark et al. 121724 (MO, no visto). E. rigidum: municipio Cajigal, Río Grande, Yaguaraparo, 19-02-2007, C. Leopardi y F. Subero 92 (IRBR). E. rostratum: municipio Cajigal, Península de Paria, 21-02-1980, J. Steyermark et al. 121468 (VEN). E. secundum: municipio Montes, Río Totucual, El Guamal, macizo montañoso del Turimiquire, (03-06)-081993, L. Cumana et al. 5011(IRBR). E. strobiliferum: municipio Benítez, Serranía La Paloma, 19-02-1980, J. Steyermark et al. 121379 (VEN). E. vincentinum: municipio Mariño, Península de Paria, Cerro de Humo, 01-03-1966, J. Steyermark 94828 (VEN). Eulophia, E. alta: municipio Arismendi, Península de Paria, entre Río Grande y Mejillones, 02-08-1961, L. Aristeguieta y G. Agostini 4792 (VEN). Gongora, G. quinquenervis: municipio Sucre, Alrededores de Los Altos de Santa Fe, feb-1994, D. Hamenstein 1112/92 (VEN). Govenia, G. utriculata: municipio Montes, Las Piedras de Cocollar, 03-04-2007, C. Leopardi et al. 136 (IRBR). Habenaria, H. alata: municipio Sucre, San Juan de Macarapana, 06-09-2006, C. Leopardi y J. Véliz 46 (IRBR). H. bractescens: municipio Montes, Cerro Grande, La Fragua, 28-09-1974, W. Lampe 18 (IRBR). H. entomantha: Sucre, sin localidad más exacta, $S / C S / N$ (IRBR). H. heptadactyla: municipio Sucre, Bella Vista, Parque Nacional Mochima, 24-08-1990, L. Cumana y J. Véliz 4418 (IRBR). H. monorrhiza: municipio Sucre, La Guayuta (Altos de Santa Fe), 27-12-1990, L. Cumana y P. Cabeza 4581 (IRBR). H. obtusa: municipio Sucre, Bella Vista, Parque Nacional Mochima, 24-08-1990, L. Cumana y J. Véliz 4472 (IRBR). H. petalodes: municipio Mariño, Península de Paria, cumbre Las Estrellas, 30-11-1979, J. Steyermark y R. Liesner 120715 (VEN). H. trifida: municipio Sucre, Cerro imposible, 07-08-2000, L. Cumana et al. 6697 (IRBR). Heterotaxis, H. discolor: municipio Mariño, Mundo Nuevo, oeste de Cerro Humo, 07-08-1966, 
J. Steyermark y M. Rabe 96146 (VEN). Houlletia, H. odoratissima: municipio Mariño, Península de Paria, Cerro Humo, 04-03-1966, J. Steyermark 95020 (VEN). Huntleya, H. lucida: municipio Valdez, Cerro Patao, norte de Puerto Hierro, S/F, J. Steyermark y G. Agostini 91387 (VEN). Isochilus, I. linearis: municipio Montes, Las Piedras de Cocollar, 02-04-2007, C. Leopardi et al. 134 (IRBR). Jacquiniella, J. globosa: municipio Montes, Cocollar, 0402-2007, L. Cumana, et al. 6978 (IRBR). J. teretifolia: municipio Montes, Cerro Turimiquire, 1945, J. Steyermark 62663 (VEN). Laelia, L. undulata: municipio Montes, Adyacencias de Cumanacoa, feb-2007, R. Coronado S/N (IRBR). Leochilus, L. labiatus: municipio Montes, Río Arriba, Las Piedras de Cocollar, 01-04-2007, C. Leopardi et al. 108 (IRBR). Lepanthes, L. pariaënsis: municipio Mariño, Península de Paria, cerca de La Tacarigua, 23-021979, J. Steyermark et al. 121640 (VEN). Lepanthes, L. pectinata: municipio Mariño, Península de Paria: Cerro de Humo, 24-02-1980, J. Steyermark y M. Rabe 96349 (VEN). L. wageneri: municipio Mariño, Península de Paria, Cerro Humo, mar-1966, J. Steyermark 94819 (VEN). Lepanthopsis, L. astrophora: municipio Mariño, Península de Paria, Las Melenas, 01-12-1979, J. Steyermark y R. Liesner 120926 (VEN). Liparis, L. nervosa subsp. nervosa: municipio Sucre, Altos de Santa Fe, 23-07-1990, L. Cumana 4133 (IRBR). Lophiaris, L. lanceana: municipio Benítez, Entre Guaraúnos y Los Ajíes, J. Steyermark et al. 121294 (VEN). L. lurida: municipio Mariño, La Montana, cerca Irapa, 23-05-1968, Carreño $S / N$ (VEN). Macroclinium, $M$. wullschlaegelianum: municipio Mariño, Península de Paria, cercanías de Manacal, S/F, J. Steyermark y R. Liesner 120628 (VEN). Masdevallia, M. irapana: Sucre, Venezuela, 05-03-1966. J. Steyermark 95079 (AMES, no visto). M. kyponantha: municipio Mariño, Península de Paria, Cerro de Humo, 05-03-1966, J. Steyermark 95079 (VEN). M. lansbergii: entre municipios Mariño y Arismendi, Península de Paria, descendiendo a Las Melenas, feb-1980, J. Steyermark et al. 121757 (VEN). Maxillaria, M. brachybulbon: municipio Mariño, Península de Paria, Cerro de Río Arriba, 09-08-1966, J. Steyermark y M. Rabe 96206 (VEN). M. melina: municipio Cajigal, Península de Paria, 21-02-1980, J. Steyermark et al. 121475 (VEN). M. nasuta: municipio Valdez, Montaña de Falcón (camino a Los Chorros), Cristobal Colón, S/F, F. Fernández 307 (VEN). M. porrecta: municipio Mariño, Península de Paria, Cerro Humo, 04-04-1966, J. Steyermark 95029-A (VEN). Microchilus, M. paleaceus: municipio Montes, Las Piedras de Cocollar, 02-04-2007, C. Leopardi et al. 133 (IRBR). M. venezuelanus: municipio Mariño, Península de Paria, carretera a El Paujil, 20-02-1980, J. Steyermark et al. 342151-VEN. (VEN). Myoxanthus, M. exasperatus: municipio Montes, Turimiquire, (25-31)-03-1995, L.
Cumana et al. 6151 (IRBR). Notylia, N. rhombilabia: Sucre, abr-1977, Carreño S/N (VEN). Octomeria, O. apiculata: municipio Valdez, Península de Paria, Cerro Patao, 19-07-1962, J. Steyermark y G. Agostini 91143 (VEN). Oeceoclades, O. maculata: municipio Benítez, Río El Pilar, Hacienda El Otro Lado, 06-05-2007, C. Leopardi y C. Malavé 152 (IRBR). Oncidium, O. klotzschianum: municipio Cajigal, Península de Paria, 21-02-1980, J. Steyermark et al. 121474 (VEN). Ornithocephalus, O. bonplandii: municipio Sucre, Altos de Santa Fé, 03-042007, Leopardi 63 (IRBR). Ornitidium, O. patulum: Entre la parte superior de Río Colorado y Cerro Turimiquire, S/F, J. Steyermark 62650 (VEN). Otoglossum, O. globuliferum: municipio Mariño, camino a Los Pocitos de Santa Isabel, Cerro Humo, 12-07-1972, G. Morillo 2591(VEN). Otoglossum, O. scansor: municipios Mariño y Arismendi, Península de Paria, 22-02-1980, J. Steyermark et al. 121542 (VEN). Pachyphyllum, P. pastii: municipio Montes, Cerro Turimiquire, 06-05-1945, J. Steyermark 62568 (VEN). Platystele, P. oxyglossa: municipio Mariño, Península de Paria, Cerro Humo, 23-02-1980, J. Steyermark et al. 121692 (VEN). Platystele stenostachya: municipio Mariño, Península de Paria, Cerro de Humo, oeste de Santa Isabel, mar-1966, J. Steyermark 94831-A (VEN). Pleurothallis, $P$. discoidea: municipio Mariño, Península de Paria, oeste de Cerro Humo, 07-08-1966, J. Steyermark y M. Rabe 96419 (VEN). P. macrophylla: municipio Mariño, Los Pocitos de Santa Isabel, Cerro de Humo, 12-07-1972, Morillo 2610 (VEN). P. pruinosa: municipio Sucre, El Guayabito, (2022)-11-1981, G. Davidse y A. González 19161 (VEN). P. revoluta: municipio Mariño, Península de Paria, cumbre de Las Estrellas, oeste de Manacal, 30-11-1979, J. Steyermark y R. Liesner 120815 (VEN). P. ruscifolia: municipio Mariño, Cerro de Humo, entre Los Pocitos y La Roma, 1108-1966, J. Steyermark y M. Rabe 96330 (VEN). P. aff. secunda: municipio Montes, Macizo montañoso del Turimiquire, fila La Pesjua, (16-20)-03-1994, L. Cumana et al. 5860 (IRBR). P. velaticaulis, municipio Montes, Las Piedras de Cocollar, macizo montañoso del Turimiquire, 23-12-2007, C. Leopardi 156 (IRBR). Polystachya, P. foliosa: municipio Cajigal, Yaguaraparo, 19-02-2007, C. Leopardi y F. Subero 97 (IRBR). Prostechea, P. cochleata: municipio Montes, Río Totucual - El Guamal, macizo Turimiquire, (03-06)-08-1993, L. Cumana et al. 5576 (IRBR). P. aemula: municipio Benítez, Río El Pilar, 06-052007, C. Leopardi y C. Malavé 145 (IRBR). P. livida: municipio Sucre, Altos de Santa Fe, 21-05-2006, C. Leopardi $S / N$ (IRBR). Psilochilus, P. modestus: municipio Mariño, Península de Paria, entre Manacal y La Cuchilla, 13-08-1966, J. Steyermark y M. Rabe 96415 (VEN). Psychopsis, P. papilio, municipio Sucre, Altos de Santa Fe, 22-01-2007, C. Leopardi 64. (IRBR). Psygmorchis, P. 
glossomystax: municipio Sucre, Altos de Santa Fe, 22-012007, C. Leopardi 61 (IRBR). P. pusilla: municipio Bolívar, Marigüitar, Río Frío, 20-02-1989, L. Cumana y P. Cabeza 3560 (IRBR). Rhetinantha, R. notylioglossa: municipio Valdez, Península de Paria, Cerro Patao, norte de Puerto Hierro, 19-07-1972, J. Steyermark y G. Agostini 91144 (VEN). Rodriguezia, R. lanceolata: municipio Benítez, Río E1 Pilar, Hacienda El Otro Lado, 06-05-2007, C. Leopardi y C. Malavé 148 (IRBR). Sauvetrea, S. aff. alpestris: municipio Montes, Macizo montañoso del Turimiquire, (25-31)-03-1995, L. Cumana et al. 6180 (IRBR). Scaphosepalum, S. breve: municipio Cajigal, Península de Paria, 21-02-1980, J. Steyermark et al. 121477 (VEN). Scaphyglottis, S. grandiflora: municipio Mariño, Península de Paria, Cerro Humo, entre El Paujil y El Brasil, 11-081966, J. Steyermark y M. Rabe 96351 (VEN). S. modesta: municipio Valdez, Península de Paria, Cerro Patao, (2526)-07-1962, J. Steyermark y G. Agostini 91393 (VEN). S. prolifera: municipio Montes, Palomar, PeriquitosTurimiquire, 10-11-1996, L. Cumana 6328 (IRBR). S. reflexa, municipio Arismendi, Península de Paria, entre La Tacarigua y las cabeceras del Río Tacarigua, 21-02-1980, J. Steyermark et al. 121465 (VEN). Sobralia, S. cf. ciliata: municipio Montes, El Guamal, fila La Pesjua, Macizo Montañoso del Turimiquire, (23-31)-03-1995, L. Cumana et al. 6208 (IRBR). S. aff. violacea: municipio Sucre, Altos de Santa Fe, 22-01-2007, Leopardi 66 (IRBR). Stelis, S. argentata: municipio Sucre, El Guayabito, (20-22)-111981, G. Davidse y A. González 19142 (VEN). S. papaquerensis: municipio Mariño, Península de Paria, camino de Los Pocitos de Santa Isabel a La Roma, 12-071972, Dumont et al. VE-7642 (VEN). S. tristyla: municipio Mariño, Península de Paria, Las Melenas, 01-12-1979, J. Steyermark y R. Liesner 120890 (VEN). Stenia, S. pallida: municipio Mariño, Península de Paria, Cerro de Humo, 1108-1966, J. Steyermark y M. Rabe 96326-A (VEN). Trichosalpinx, T. ciliaris: municipio Cajigal, Península de Paria, entre E1 Paujil y El Brasil, 21-02-1980, J. Steyermark et al. 121507 (VEN). T. intricata: municipios Mariño y Arismendi, Península de Paria, Las Melenas, 24-02-1980, J. Steyermark et al. 121757 (VEN). Triphora, T. gentianoides: municipio Valdez, Península de Paria, Cerro Patao, 20-07-1962, J. Steyermark y G. Agostini 67582 (VEN). Trizeuxis, T. falcata: municipio Cajigal, Yaguaraparo, 19-02-2007, C. Leopardi y F. Subero 82 (IRBR). Vanilla, V. pompona: municipio Sucre, Sabilar, nov-1984, Cumana 2596 (IRBR). Wullschlaegelia, W. calcarata: municipio Valdez, Península de Paria, Cerro Patao, (25-26)-07-1962, J Seyermark y G. Agostini 91334 (VEN). Xylobium, X. pallidiflorum: municipio Benítez, Serranía de La Paloma, 19-02-1980, J. Steyermark et al. 121345 (VEN). 
LANKESTERIANA 